\title{
Avaliação das dimensões da sede: revisão integrativa*
}

\author{
Pamela Rafaela Martins ${ }^{1}$, Lígia Fahl Fonseca ${ }^{2}$
}

\author{
* Artigo extraído da dissertação de \\ mestrado intitulada "Elaboração e \\ validação de uma Escala de Desconforto da \\ Sede Perioperatória", apresentada ao \\ Programa de Pós-Graduação em \\ Enfermagem da Universidade Estadual de \\ Londrina, 2016. \\ ${ }^{1}$ Enfermeira, Mestre em Enfermagem. \\ Professora da Faculdade Integradas de \\ Cassilândia. Cassilândia, MS, Brasil. E-mail: \\ pammartins253@gmail.com. \\ ${ }^{2}$ Enfermeira, Doutora em Enfermagem \\ Saúde do Adulto. Professora Associada da \\ Universidade Estadual de Londrina. \\ Londrina, PR, Brasil. E-mail: \\ ligiafahl@gmail.com.
}

Recebido: 15/03/2016.

Aceito: 26/01/2017.

Publicado: 15/05/2017.

\section{Como citar esse artigo}

Martins PR, Fonseca LF. Avaliação das dimensões da sede: revisão integrativa. Rev. Eletr. Enf. [Internet]. 2017 [acesso em: ____19:a09. Disponível em: http://dx.doi.org/10.5216/ree.v19.40288.

\begin{abstract}
RESUMO
A sede é um sintoma multifatorial e subjetivo cuja mensuração requer múltiplos instrumentos. O objetivo deste estudo foi identificar as dimensões de avaliação da sede e os instrumentos de mensuração utilizados. Incluíram-se artigos publicados entre 2005 a 2015 das bases de dados Lilacs, PubMed e SciELO. A amostra final foi de 18 artigos que evidenciaram as dimensões da sede: intensidade, frequência e desconforto. Também se avaliou a xerostomia e sua intensidade. Essas dimensões foram identificadas por Escalas Visuais Analógicas, Escalas Verbais Numéricas, Escalas de Faces e Escalas Likert, empregadas no Inventário de Sede, Inventário de Xerostomia e Escala de Desconforto da Sede. Avaliou-se a sede principalmente em pacientes dialíticos e internados em unidade de terapia intensiva. Embora a sede seja um sintoma, sua avaliação concentra-se sobretudo na intensidade e é realizada em populações específicas.
\end{abstract}

Descritores: Sede; Avaliação; Escalas; Enfermagem.

\section{INTRODUÇÃO}

A regulação do consumo de água é controlada pela sede. Embora seja regulado por mecanismos fisiológicos, o comportamento de ingestão de líquidos é influenciado por hábitos, costumes e rituais sociais do indivíduo ${ }^{(1)}$. O papel da sede na homeostasia dos fluidos implica uma rede de complexos processos neurais e hormonais, em resposta a algum desequilíbrio na relação água e sódio no organismo ${ }^{(2-3)}$.

Para os pacientes nos ambientes de saúde, a sede é um dos sintomas mais prevalentes e desconfortáveis, podendo sobrepujar todas as outras sensações ${ }^{(1,4-5)}$. Pacientes em unidade de terapia intensiva (UTI), dialíticos e aqueles em pré e pós-operatório descrevem-na como uma experiência desagradável, intensa, que provoca estresse e desespero ${ }^{(3,6-8)}$. Para retratar a experiência da sede, chegam a 
empregar figuras alusivas à loucura e até à morte ${ }^{(5)}$. Sua prevalência pode alcançar até $75 \%$ no pós-operatório imediato $^{(5,9)}$.

Define-se sintoma como uma experiência subjetiva, responsável por alterações no funcionamento biopsicossocial, nas sensações e até mesmo na cognição do indivíduo ${ }^{(10)}$. A sede é um sintoma multifatorial, subjetivo e complexo; sua identificação e mensuração devem ser criteriosas e detalhadas, objetivando caracterizar suas dimensões, para possibilitar ações direcionadas para o manejo e alívio.

Pode-se avaliar a sede por exames laboratoriais, pela osmolaridade do sangue e pela dosagem do hormônio antidiurético (ADH). Avanços tecnológicos identificam a sede pelo mapeamento das áreas cerebrais envolvidas, por técnicas de indução e análise concomitante de imagens obtidas com tomografia e ressonância ${ }^{(11)}$.

Os métodos com base na neurofisiologia da sede permitem identificá-la, não sendo, entretanto, únicos, já que a sede é um sintoma que pode vir a ser uma experiência intensa e desconfortável, caracterizada pela subjetividade e individualidade, tornando sua avaliação um desafio. Nesse contexto, o autorrelato configura-se um balizador para a mensuração ${ }^{(1,3-4)}$.

Na prática clínica, não se avalia a sede de forma intencional e sistematizada. Os registros de sua avaliação são escassos - quando não inexistentes - e contemplam sobretudo a intensidade. A avaliação da sede deve abranger sua multifatoriedade, dentro das dimensões referentes a: prevalência, incidência, frequência, duração e desconforto ${ }^{(2-4)}$.

O presente estudo se justificou pela necessidade de compreender como a sede, em razão de sua magnitude e complexidade, está sendo avaliada. Esta revisão integrativa objetivou identificar as dimensões de avaliação da sede e os instrumentos de mensuração utilizados. O desenvolvimento de futuras pesquisas, a disponibilização do compilado de conhecimento sobre estratégias possíveis para mensuração de uma necessidade humana básica frequentemente subvalorizada e subavaliada ${ }^{(12)}$ certamente contribuirá para sua implantação na prática clínica.

\section{MÉTODOS}

Trata-se de uma revisão integrativa de literatura, método que reúne e sintetiza resultados de pesquisas experimentais e não experimentais de maneira sistemática e organizada, a partir da delimitação de um tema ${ }^{(13)}$.

A seguinte pergunta norteou a investigação: Quais são as evidências científicas disponíveis na literatura sobre avaliação das dimensões da sede? A pesquisa dos artigos baseou-se nos Descritores em Ciências da Saúde: sede-thirst; xerostomia-xerostomia; privação de água-water deprivation; desidrataçãodehydration; avaliação-assessment; escalas-scales e enfermagem-nursing. Os descritores foram utilizados de forma isolada e combinados entre si.

Para a escolha dos artigos, selecionaram-se os bancos de dados Scientific Eletronic Library Online (SciELO), Literatura Latino-Americana e do Caribe em Ciências da Saúde (Lilacs) e US National Library of 
Medicine (PubMed). Selecionados os estudos primários, buscaram-se também os artigos que respondiam à pergunta da revisão mencionados nas referências dos textos escolhidos. Fez-se a busca dos artigos em maio e junho de 2015.

Para selecionar as publicações incluídas na pesquisa, adotaram-se as recomendações do PRISMA (Figura 1). Os critérios de inclusão foram: artigos em português, inglês e espanhol, publicados entre 2005 e 2015 e disponíveis na íntegra.

Na primeira análise, com base na leitura do título e do resumo das publicações, excluíram-se os artigos que não abordavam avaliação da sede. Na segunda análise, pela leitura do artigo na íntegra, excluíram-se aqueles que não contemplaram a mensuração de dimensões da sede por escalas.

Em conformidade com o instrumento elaborado pelos autores, extraíram-se dos estudos informações referentes a: autoria, ano de publicação, título, método, objetivos, dimensão da sede abordada na avaliação e resultados do estudo.

\section{RESULTADOS}

Foram analisados os títulos e resumos de 46 artigos científicos, obtidos a partir dos bancos de dados e referências, dos quais 22 foram eliminados por não contemplarem a temática em estudo. Após uma segunda análise do artigo na íntegra, a amostra final foi composta por 18 artigos, cinco dos quais estavam no Lilacs, cinco no PubMed, e oito foram obtidos da análise das referências dos artigos inicialmente selecionados nos bancos de dados. Os artigos encontrados na SciELO se repetiram ou não contemplaram a temática em estudo (Figura 1). Um artigo foi publicado em português, os demais em inglês. Dos 18 artigos incluídos na amostra final, 11 (61,1\%) foram publicados nos Estados Unidos, quatro (22,2\%) na Inglaterra; França, Nigéria e Brasil produziram um $(5,6 \%)$ cada, sendo 2010 e 2014 os anos em que mais se publicou.

Predominaram ensaios clínicos randomizados (33\%). A avaliação da sede ocorreu principalmente em pacientes com doença renal crônica submetidos à hemodiálise, constando em sete artigos ${ }^{(14-20)}$; realizaramse quatro estudos com pacientes em UTI ${ }^{(21-24)}$; um com pacientes adultos em pós-operatório imediato ${ }^{(6)}$; um com sujeitos com anemia falciforme ${ }^{(25)}$ e um com pacientes que se recuperaram de um estado de choque séptico ${ }^{(26)}$. Dois estudos envolveram sujeitos adultos sem nenhuma condição patológica ${ }^{(27-28)}$, e outros dois foram realizados com crianças $^{(29-30)}$.

A avaliação das dimensões da sede foi feita para identificar sobretudo intensidade $(15)^{(9,16-18,20-30)}$, desconforto $(4)^{(17,22-24)}$ e frequência do sintoma (3) $)^{(14-16)}$, assim como para avaliar a xerostomia e sua intensidade $(5)^{(14-16,18-19)}$. Outras finalidades também foram evidenciadas: correlacionar a sede com ganho de peso dialítico $(3)^{(14,18-19)}$, redução de secreção salivar $(2)^{(15,18)}$ e qualidade de vida de pacientes em hemodiálise (7) ${ }^{(14-20)}$; avaliar a sede após alguma intervenção - como água em temperatura ambiente e lascas de gelo $(1)^{(9)}$, gomas de mascar e substitutos da saliva $(1)^{(14)}-$, um pacote de intervenções composto por swabs orais, sprays de água, hidratante labial com mentol $(1)^{(23)}$, e administração de pilocarpina (1) ${ }^{(18)}$; por fim, comparar escores de sede com o conteúdo gástrico residual em crianças $(1)^{(29)}$ e com áreas cerebrais ativadas 
pela sede e sua saciedade $(1)^{(27)}$.

As escalas utilizadas para a avaliação da sede foram: Escala Visual Analógica (EVA), Escala Verbal Numérica (EVN), Escala de Faces (EF) e as escalas Likert. Abordou-se a dimensão intensidade da sede na utilização da EVA em oito estudos ${ }^{(16-18,25-28,30)}$, da EVN em seis ${ }^{(9,20-21,23-24,29)}$, e da EF em dois ${ }^{(29-30)}$. Em um único estudo $^{(22)}$ se adotou escala tipo Likert de três pontos para avaliação da intensidade e desconforto provocado pela sede.

A EVA, EVN e EF, em conjunto com as Escalas de Descritores Verbais (EDV), são instrumentos de avaliação válidos e fidedignos para a mensuração da dor em diferentes populações ${ }^{(31-34)}$. Esses quatro tipos de escalas foram adaptadas para a avaliação da sede. A EDV não constou em nenhuma publicação da amostra final.

A EVA consiste em uma linha de 10 centímetros ou 100 milímetros, com as extremidades representando os extremos de sede, e o restante da linha, níveis intermediários ${ }^{(34)}$. 0 respondente deve marcar o ponto da linha que representa a sua sede; consequentemente deve haver contato visual do paciente com a escala, exigindo dele boa acuidade visual e cognitiva para classificar sua sede ${ }^{(33-34)}$.

A EVN, entretanto, permite que o sujeito classifique sua sede em uma escala de zero a dez, em que zero corresponde a "sem sede", e dez, "a pior sede possível”(34-35). A aplicação da EVN não requer obrigatoriamente contato visual do respondente ${ }^{(33)}$.

A adoção de EF ocorreu em estudos que incluíam a identificação da intensidade da sede em crianças. As $E F$, inicialmente desenvolvidas para crianças, vêm sendo utilizadas em adultos com dificuldade de comunicação ${ }^{(36)}$.

Em um estudo exploratório ${ }^{(17)}$, usou-se escala Likert de cinco pontos, com validade e fidedignidade, para a identificação do desconforto provocado pela sede em pacientes submetidos à hemodiálise, evidenciando altos escores de sede. A EVN também avaliou a dimensão desconforto em dois estudos ${ }^{(23-24)}$. Em três pesquisas ${ }^{(14-16)}$, mensurou-se a xerostomia por escala Likert de cinco pontos, empregada no Inventário de Xerostomia (Xerostomia Inventory); a identificação de sua intensidade se deu pela EVA em três publicações ${ }^{(16,18-19)}$. O domínio frequência esteve presente em três estudos ${ }^{(14-16)}$, no Inventário de Sede (Dialysis Thirst Inventory) direcionado para pacientes em hemodiálise, com itens referentes à periodicidade de acometimento do sintoma, correlacionado com as sessões de hemodiálise.

Seis publicações ${ }^{(14-17,29-30)}$ adotaram mais de um instrumento para mensurar as dimensões da sede.

o Quadro 1 apresenta as principais informações extraídas das publicações tabuladas. 
Figura 1: Fluxo do processo de seleção dos estudos para a revisão integrativa de literatura, elaborado com base nas recomendações PRISMA. Londrina, PR, Brasil, 2015.

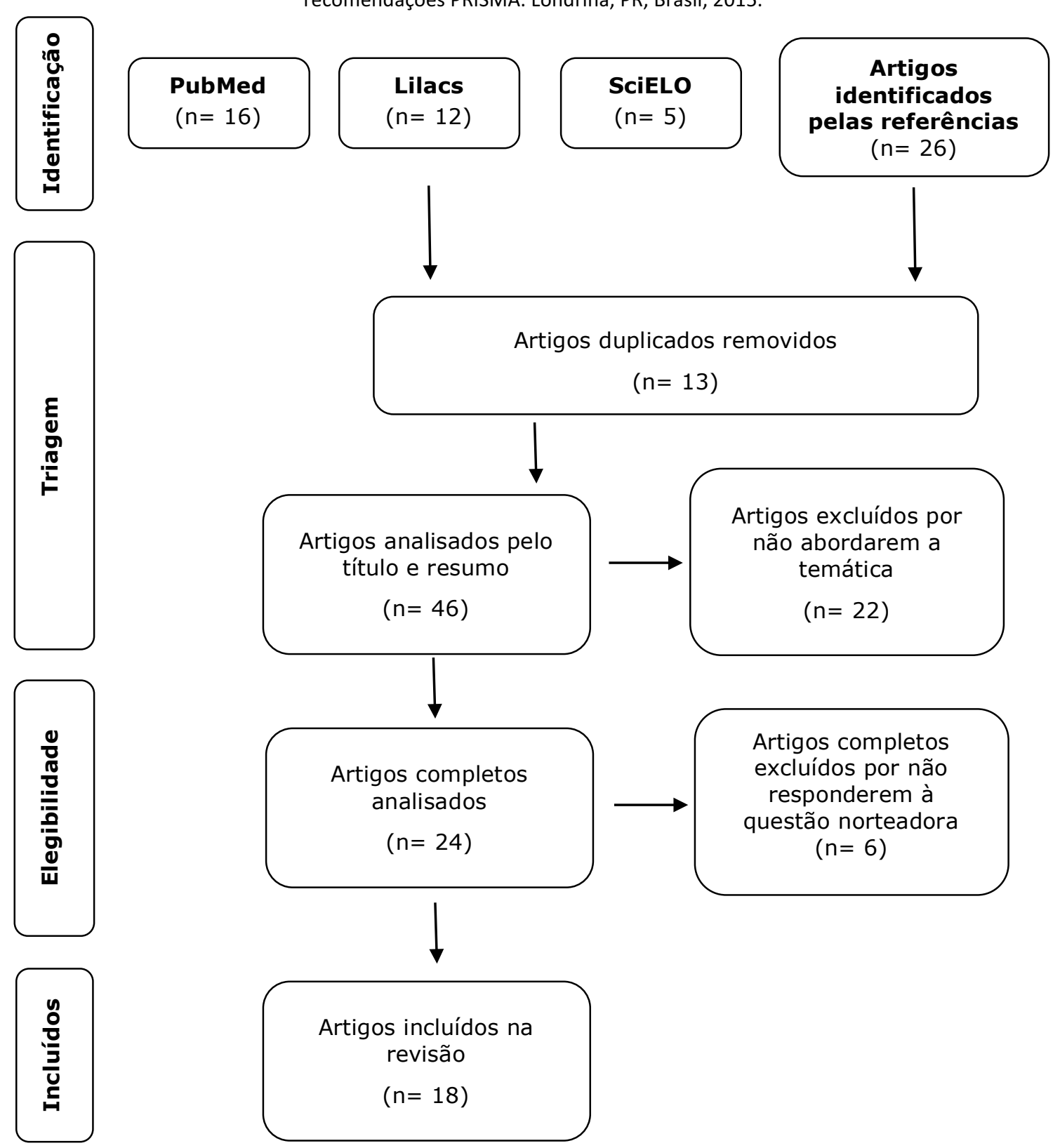


Quadro 1: Publicações sobre avaliação das dimensões da sede, segundo autores, ano, método, objetivo, dimensão da sede avaliada (2005 a 2015$)$. Londrina, PR, Brasil, 2015.

\begin{tabular}{|c|c|c|}
\hline Autores/ Ano & Método e Objetivo & Dimensão da Sede Avaliada/Instrumento utilizado \\
\hline Aroni, Nascimento, Fonseca $(2012)^{(9)}$ & $\begin{array}{l}\text { Estudo quase experimental; avaliar estratégias para alívio da sede em } 90 \text { pacientes no pós-operatório } \\
\text { imediato. }\end{array}$ & $\begin{array}{l}\text { Avaliação da intensidade da sede por Escala Verbal } \\
\text { Numérica (0 a 10) }\end{array}$ \\
\hline \multirow{2}{*}{$\begin{array}{l}\text { Bots, Brand, Veerman, Korevaar, } \\
\text { Valentijn-Benz, Bezemer et al. } \\
\qquad(2005)^{(14)}\end{array}$} & \multirow{2}{*}{$\begin{array}{l}\text { Ensaio clínico randomizado; investigar o potencial efeito da goma de mascar sem açúcar e um } \\
\text { substituto da saliva na xerostomia, sede e ganho de peso em } 65 \text { pacientes com doença renal crônica } \\
\text { em estágio final submetidos à hemodiálise. }\end{array}$} & $\begin{array}{l}\text { Avaliação da frequência da sede por Escala Likert de } \\
\text { cinco pontos - Inventário de Sede }\end{array}$ \\
\hline & & $\begin{array}{l}\text { Avaliação da xerostomia por Escala Likert de cinco } \\
\text { pontos - Inventário de Xerostomia }\end{array}$ \\
\hline \multirow{2}{*}{$\begin{array}{l}\text { Bots, Brand, Poorterman, } \\
\text { Amerongen, Valentijn-Benz, Veerman } \\
\text { et al. }(2007)^{(15)}\end{array}$} & \multirow{2}{*}{$\begin{array}{l}\text { Estudo observacional longitudinal; comparar aspectos da cavidade oral, secreção de saliva, xerostomia } \\
\text { em } 43 \text { indivíduos com doença renal crônica depois de transplante renal. }\end{array}$} & $\begin{array}{l}\text { Avaliação da frequência da sede por Escala Likert de } \\
\text { cinco pontos - Inventário de Sede }\end{array}$ \\
\hline & & $\begin{array}{l}\text { Avaliação da xerostomia por Escala Likert de cinco } \\
\text { pontos - Inventário de Xerostomia }\end{array}$ \\
\hline \multirow{3}{*}{ Fan, Zhang, Luo, Niu, Gu (2013) ${ }^{(16)}$} & \multirow{3}{*}{$\begin{array}{l}\text { Ensaio clínico randomizado; investigar o significado clínico e fatores relacionados com a sede e } \\
\text { xerostomia em } 42 \text { pacientes em hemodiálise. }\end{array}$} & $\begin{array}{l}\text { Avaliação da intensidade da sede e xerostomia por } \\
\text { Escala Visual Analógica (0 a 10) }\end{array}$ \\
\hline & & $\begin{array}{l}\text { Avaliação da frequência da sede por Escala Likert de } \\
\text { cinco pontos - Inventário de Sede }\end{array}$ \\
\hline & & $\begin{array}{l}\text { Avaliação da xerostomia por Escala Likert de cinco } \\
\text { pontos - Inventário de Xerostomia }\end{array}$ \\
\hline \multirow{2}{*}{ Porcu, Fanton, Zampieron (2007) ${ }^{(17)}$} & \multirow{2}{*}{$\begin{array}{l}\text { Estudo correlacional; descrever as percepções de sede e correlacionar com os sintomas vivenciados } \\
\qquad \text { por } 107 \text { pacientes submetidos à hemodiálise. }\end{array}$} & $\begin{array}{l}\text { Avaliação da intensidade da sede por Escala Visual } \\
\text { Analógica (0 a 10) }\end{array}$ \\
\hline & & $\begin{array}{l}\text { Avaliação do desconforto da sede por Escala Likert } \\
\text { de cinco pontos - Escala de Desconforto da Sede }\end{array}$ \\
\hline $\begin{array}{l}\text { Sung, Kuo, Guo, Chuang, Lee, Huang } \\
\qquad(2005)^{(18)}\end{array}$ & $\begin{array}{l}\text { Ensaio clínico randomizado; determinar se a redução na secreção de saliva contribui para o aumento } \\
\text { da sede e do ganho de peso em } 90 \text { pacientes submetidos à hemodiálise. Avaliar a ação da pilocarpina } \\
\text { em reduzir a sensação de boca seca. }\end{array}$ & $\begin{array}{l}\text { Avaliação da intensidade de sede e xerostomia por } \\
\text { Escala Visual Analógica ( } 0 \text { a 10) }\end{array}$ \\
\hline $\begin{array}{l}\text { Sung, Kuo, Guo, Chuang, Lee, Huang } \\
(2006)^{(19)}\end{array}$ & $\begin{array}{l}\text { Estudo prospectivo de coorte; investigar a relação da xerostomia e ganho de peso dialítico em } 116 \\
\text { pacientes sem diabetes mellitus e em } 68 \text { diabéticos que fazem hemodiálise. }\end{array}$ & $\begin{array}{l}\text { Avaliação da intensidade da xerostomia por Escala } \\
\text { Visual Analógica ( } 0 \text { a } 10)\end{array}$ \\
\hline Yang, Yates, Chin, Kao (2010) $)^{(20)}$ & $\begin{array}{l}\text { Ensaio clínico randomizado com cegamento simples; avaliar a eficácia da acupuntura não invasiva em } \\
\text { estimular o aumento na secreção salivar e reduzir a sede em } 28 \text { pacientes em hemodiálise. }\end{array}$ & $\begin{array}{l}\text { Avaliação da intensidade da sede por Escala Verbal } \\
\text { Numérica ( } 0 \text { a } 10)\end{array}$ \\
\hline Li, Puntillo (2006) & $\begin{array}{l}\text { Estudo-piloto prospectivo; levantamento da prevalência e intensidade de nove sintomas vivenciados } \\
\qquad \text { por } 15 \text { pacientes em UTI que estavam entubados. }\end{array}$ & $\begin{array}{l}\text { Avaliação da intensidade da sede por Escala Verbal } \\
\text { Numérica ( } 0 \text { a } 10)\end{array}$ \\
\hline $\begin{array}{l}\text { Puntillo, Arai, Cohen, Gropper, } \\
\text { Neuhaus, Paul (et al. 2010) }\end{array}$ & Estudo prospectivo e observacional; avaliação dos sintomas vivenciados por 171 pacientes em UTI. & $\begin{array}{l}\text { Avaliação da intensidade e desconforto da sede por } \\
\text { Escala tipo Likert de três pontos }\end{array}$ \\
\hline $\begin{array}{l}\text { Puntillo, Arai, Cooper, Stotts, Nelson } \\
\qquad(2014)^{(23)}\end{array}$ & $\begin{array}{l}\text { Ensaio clínico randomizado; testar um pacote de intervenções para reduzir a intensidade e } \\
\text { desconforto da sede e boca seca em } 252 \text { pacientes de UTI. }\end{array}$ & $\begin{array}{l}\text { Avaliação da intensidade e desconforto da sede por } \\
\text { Escala Verbal Numérica (0 a 10) }\end{array}$ \\
\hline $\begin{array}{l}\text { Stotts, Arai, Cooper, Nelson, Puntillo } \\
\qquad(2015)^{(24)}\end{array}$ & $\begin{array}{l}\text { Estudo descritivo transversal; identificar preditores da presença, intensidade e desconforto da sede } \\
\qquad \text { em } 353 \text { pacientes de UTI. }\end{array}$ & $\begin{array}{l}\text { Avaliação da intensidade e desconforto da sede por } \\
\text { Escala Verbal Numérica (0 a 10) }\end{array}$ \\
\hline
\end{tabular}

Rev. Eletr. Enf. [Internet]. 2017 [acesso em:___/_];19:a09. Disponível em: http://dx.doi.org/10.5216/ree.v19.40288. 


\begin{tabular}{|c|c|c|}
\hline Autores/ Ano & Método e Objetivo & Dimensão da Sede Avaliada/Instrumento utilizado \\
\hline $\begin{array}{l}\text { Ozoene, Enosolease, Ajayi, Agoreyo, } \\
\text { Obika (2009) }\end{array}$ & $\begin{array}{l}\text { Estudo transversal analítico; examinar a percepção da sede em } 20 \text { voluntários com e sem a doença } \\
\text { falciforme durante } 13 \text { horas de privação de água. }\end{array}$ & $\begin{array}{l}\text { Avaliação da intensidade da sede por Escala Visual } \\
\text { Analógica (0 a 10) }\end{array}$ \\
\hline $\begin{array}{l}\text { Siami, Polito, Porcher, Hissem, } \\
\text { Blanchard, Boucly et al. }(2013)^{(26)}\end{array}$ & $\begin{array}{l}\text { Estudo prospectivo de intervenção; descrever a avaliação da mudança de vasopressina (ADH) no } \\
\text { plasma após infusão salina em } 30 \text { pacientes que se recuperaram de choque séptico. }\end{array}$ & $\begin{array}{l}\text { Avaliação da intensidade da sede por Escala Visual } \\
\text { Analógica ( } 0 \text { a 10) }\end{array}$ \\
\hline $\begin{array}{l}\text { Farrel, Zamarripa, Shade, Phillips, } \\
\text { McKinley, Fox et al. (2008) }\end{array}$ & $\begin{array}{l}\text { Estudo experimental; investigar a geração da sede em } 12 \text { indivíduos idosos e } 10 \text { jovens por meio de } \\
\text { infusão de solução salina hipertônica. }\end{array}$ & $\begin{array}{l}\text { Avaliação da intensidade da sede por Escala Visual } \\
\text { Analógica (0 a 10) }\end{array}$ \\
\hline $\begin{array}{l}\text { Pross, Demazières, Girard, Barnouin, } \\
\text { Metzger, Klein et al. (2014) }\end{array}$ & $\begin{array}{l}\text { Estudo quase experimental; avaliar os efeitos no aumento e redução da ingestão de água, no estado } \\
\text { de humor e em algumas sensações em } 52 \text { voluntários. }\end{array}$ & $\begin{array}{l}\text { Avaliação da intensidade da sede por Escala Visual } \\
\text { Analógica ( } 0 \text { a 10) }\end{array}$ \\
\hline \multirow{2}{*}{$\begin{array}{l}\text { Buehrer, Hanke, Klaghofer, Fruehauf, } \\
\qquad \text { Weiss, Schmitz }(2014)^{(29)}\end{array}$} & \multirow{2}{*}{$\begin{array}{l}\text { Estudo experimental; comparar pontuações de fome e sede com imagens de ressonância magnética } \\
\text { do conteúdo gástrico residual, em } 30 \text { crianças voluntárias de } 6 \text { a } 12 \text { anos. }\end{array}$} & $\begin{array}{l}\text { Avaliação da intensidade da sede por Escala Verbal } \\
\qquad \text { Numérica }(0 \text { a 10) }\end{array}$ \\
\hline & & $\begin{array}{l}\text { Avaliação da intensidade da sede por Escala de } \\
\text { Faces }\end{array}$ \\
\hline \multirow{2}{*}{$\begin{array}{l}\text { Klemetti, Kinnunen, Suominen, Antila, } \\
\text { Vahlberg, Grenman et al. }(2010)^{(30)}\end{array}$} & \multirow{2}{*}{$\begin{array}{l}\text { Ensaio clínico randomizado; analisar se o aconselhamento pré-operatório nutricional face a face sobre } \\
\text { o jejum pode melhorar as respostas da fome e sede em } 116 \text { crianças, de } 4 \text { a } 10 \text { anos, no pós- } \\
\text { operatório de cirurgia de amigdalectomia. }\end{array}$} & $\begin{array}{l}\text { Avaliação da intensidade da sede por Escala Visual } \\
\text { Analógica (0 a 10) }\end{array}$ \\
\hline & & $\begin{array}{l}\text { Avaliação da intensidade da sede por Escala de } \\
\text { Faces }\end{array}$ \\
\hline
\end{tabular}




\section{DISCUSSÃO}

A sede, é descrita como o desejo de beber água, trata-se de um sintoma subjetivo e multifatorial, influenciado por aspectos culturais, sociais, ambientais, e pelos hábitos e costumes do indivíduo ${ }^{(1,4)}$. Mensurá-la, portanto, torna-se um desafio pela complexidade em se avaliar suas múltiplas dimensões e aspectos. Este estudo contribui para o aprofundamento na compreensão do sintoma sede por identificar e compilar as dimensões de avaliação e os instrumentos de mensuração utilizados para avaliar, dentro e fora de ambientes hospitalares.

Nas publicações analisadas, predominou a avaliação da dimensão intensidade da sede. Identificou-se a intensidade pela aplicação de escalas extensivamente utilizadas para a avaliação da dor: EVA, EVN e EF. A EVA também avaliou intensidade de xerostomia.

O comportamento que resulta na ingestão de água se deve principalmente a alterações volêmicas e no equilíbrio hidroeletrolítico ${ }^{(1)}$. Devido à necessidade de avaliar alterações osmóticas por métodos não invasivos, realizaram-se ensaios clínicos para investigar a aplicabilidade e a sensibilidade da EVA e EVN na avaliação da sede, correlacionando com os resultados de exames laboratoriais. Evidenciou-se correlação significativa de altos escores de sede com a alta osmolaridade sanguínea em 10 publicações; em oito, encontrou-se correlação com altos níveis do $A D H^{(2)}$. A EVA e EVN mostram-se, portanto, na prática clínica, como um método simples e indicativo das alterações osmóticas, podendo auxiliar a equipe de saúde na mensuração da sede.

A gênese da sede, além de englobar mecanismos fisiológicos, sofre ação de fatores neuronais, hormonais, comportamentos aprendidos, como os sinais referentes à boca e garganta secas e à sensação de estômago cheio ${ }^{(1,24,37)}$. Não obstante, diversos guidelines de associações científicas, dentre elas a Associação Norte-Americana de Diagnósticos de Enfermagem (NANDA), abordam a sede exclusivamente na perspectiva do equilíbrio e desequilíbrio de fluidos. Todavia tanto os mecanismos que deflagram quanto os que mitigam a sede envolvem complexos processos de interação com o meio ambiente, processos de saúde e doença, hábitos pessoais, entre outros. Dessa forma, a identificação desses componentes é importante para a implementação de ações para o manejo da sede ${ }^{(3-4,37)}$. A sede é expressa através de múltiplos atributos ou sinais, alguns dos quais como saliva grossa, lábios ressecados e língua grossa, que precisam ser levados em consideração quando se tem como objetivo mensurar a sede.

Os resultados do presente estudo indicam que as dimensões da sede identificadas são consideradas de forma isolada ou independente nos instrumentos de mensuração, com foco predominante na intensidade e no sintoma relacionado com o ressecamento das mucosas e em populações específicas, como pacientes submetidos à hemodiálise.

O ressecamento da cavidade oral é descrito como o atributo mais desagradável e o mais frequentemente descrito pelos indivíduos experienciando sede ${ }^{(1)}$. Dessa forma, instrumentos foram desenvolvidos com a finalidade específica de mensurá-lo.

O Inventário de Xerostomia (Xerostomia Inventory) é um instrumento validado e confiável testado com 
idosos $^{(38)}$, todavia é usado em pacientes com doença renal crônica submetidos à hemodiálise, para identificar a frequência da sede, em conjunto com o também validado e confiável Inventário de Sede (Dialysis Thirst Inventory) $)^{(39)}$.

O Inventário de Xerostomia abrange 11 itens; o da sede, sete. Ambos consistem em escala Likert de cinco pontos, em que um corresponde a "nunca" e cinco "com frequência", em relação à presença do item em avaliação ${ }^{(38-39)}$. Um escore final de 11 no Inventário de Xerostomia significa que "a boca não está seca", enquanto 55 indica que "a boca está extremamente seca"(38). Escore final de sete no Inventário de Sede corresponde a "sem sede"; de 35, a "muita sede"(39).

O atributo boca seca, embora um balizador para a percepção da sede, não deve ser o único determinante na caracterização e manejo da sede. O desconforto provoca alterações que ultrapassam os limites físicos, abrangendo a dimensão psicológica, social, espiritual e até ambiental da pessoa ${ }^{(40)}$. Dessa forma é importante mensurar não somente o atributo objetivo da sede, mas também o desconforto que daí decorre.

O desconforto da sede é o grau em que o indivíduo se incomoda com ela ou com um desconforto associado. Para avaliar o desconforto provocado pela sede em pacientes dialíticos, elaborou-se uma escala que passou pelo processo de validação. A Escala de Desconforto da Sede também consiste em uma escala Likert de cinco pontos, composta por seis itens, cujo escore de um corresponde a "extrema discordância", e cinco a "extrema concordância" com o item em avaliação(41).

Em um estudo desenvolvido com pacientes de UTI, mensurou-se, por duas escalas do tipo Likert de três pontos, o desconforto e a intensidade dos sintomas - entre eles a sede - vivenciados pelos pacientes. Na avaliação do desconforto, respondia-se "não muito desconfortável", "moderado desconforto" e "muito desconfortável”(22). A avaliação era de forma global, sem explicitar os componentes e dimensões que interferem no desconforto do paciente. Para a intensidade da sede, os pacientes a classificavam em "média", "moderada" e "severa" (22).

Esses instrumentos são de fato válidos e fidedignos para avaliar a xerostomia, a sede e o desconforto que ela provoca em pacientes com condições clínicas crônicas, as quais os predispõem ao desenvolvimento da sede. Entretanto, por sua extensão, podem ser de difícil aplicação a alguns grupos de pacientes, como os cirúrgicos em recuperação anestésica.

Assim, mesmo tendo sido identificadas escalas que avaliem as dimensões da sede, os estudos são escassos, incipientes e com abordagem predominante da intensidade. Estão voltados para pacientes submetidos à sessões de hemodiálise e internados em UTI, tendo sido encontrado um único estudo que avaliou a sede em pacientes cirúrgicos ${ }^{(9)}$.

Os pacientes cirúrgicos estão expostos a uma confluência de fatores que resultam em sede: jejum préoperatório, intubação, medicamentos utilizados para 0 ato anestésico-cirúrgico, sangramento intraoperatório e oxigenoterapia prolongada ${ }^{(4,9)}$. Em decorrência desses fatores, que os predispõem ao desenvolvimento da sede, é real e evidente a demanda por instrumentos que explorem e caracterizem não 
somente a intensidade da sede, mas também as diversas dimensões do desconforto experienciadas pelos pacientes no período perioperatório. Constatou-se nos estudos selecionados que a avaliação sobre o desconforto não discrimina exatamente os componentes periféricos que o provocam, como boca seca, lábios rachados e saliva grossa.

A sede é um sintoma prevalente em diversos cenários da prática clínica, sendo causadora de intenso desconforto em pacientes em hemodiálise ${ }^{(41)}, \mathrm{UTI}^{(3,7)}$, crianças $^{(42)}$ e no paciente cirúrgico ${ }^{(5,9)}$. Pacientes relatam sentimentos de desespero e fazem até mesmo alusão à morte quando são encorajados a descrever sua vivência com a sede ${ }^{(5)}$.

No entanto, a valorização desse sintoma pela equipe ${ }^{(12)}$, o uso de instrumentos que permitam sua mensuração e estratégias para tratamento são ainda insuficientes. Uma melhor caracterização da sede vivenciada pelo paciente e a abordagem intencional de mais de uma dimensão durante a avaliação podem ser o início para compreender e tratar este sintoma de modo mais efetivo.

\section{CONCLUSÃO}

Identificar e mensurar a sede e seu desconforto são os primeiros passos para ampliar a conscientização da equipe de saúde visando à utilização de medidas seguras e eficazes para minorar a sede do paciente na prática clínica.

Como resultado dessa revisão, identificou-se que as dimensões da sede - intensidade, frequência e desconforto - foram identificadas por Escala Visual Analógica, Escala Verbal Numérica e Escala de Faces, extensivamente usadas para a avaliação do sintoma dor, e pelo Inventário de Sede, Inventário de Xerostomia e Escala de Desconforto da Sede. Evidenciou-se que, além de não englobar todas as dimensões da sede, os instrumentos de avaliação existentes direcionam-se para populações específicas, como pacientes em hemodiálise e em UTI, e com foco principal na mensuração da intensidade.

Dessa forma, não se valoriza ainda a multifatoriedade causal e de repercussões para o indivíduo que experiencia a sede, uma vez que os instrumentos existentes visam à identificação de componentes isolados desse complexo sintoma. Dentre as limitações do estudo estão as diferentes metodologias utilizadas nas pesquisas encontradas, já que nem todos os instrumentos descritos passaram por processo de validação, o que dificultou a comparação entre os resultados.

Novos estudos devem ser conduzidos para a elaboração e validação de instrumentos que compreendam a avaliação da sede em sua multifatoriedade e, sobretudo, subjetividade, assim como as repercussões para o indivíduo que a experiencia.

\section{REFERÊNCIAS}

1. Leiper J. Thirst [Internet]. In: Caballero B, Allen L, Prentice A, editors. Encyclopedia of human nutrition. Vol $4.2^{\text {nd }}$ ed. Oxford, England: Elsevier Ltd/Academic Press; 2005 [acesso em: 2 out. 2015]. p. 278-86. Disponível em:

http://content.yudu.com/Library/A17k4j/EncyclopediaofHumanN/resources/1969.htm. 
2. Arai SR, Butzlaff A, Stotts NA, Puntillo KA. Quench the thirst: lessons from clinical thirst trials. Biol Res Nurs [Internet]. 2014 Oct [acesso em: 19 nov. 2015]; 16(4):456-66. Disponível em:

http://www.ncbi.nlm.nih.gov/pubmed/24136996.

3. Arai S, Stotts N, Puntillo K. Thirst in critically ill patients: from physiology to sensation. Am J Crit Care [Internet]. 2013 Jul [acesso em: 2 out. 2015]; 22(4):328-36. Disponível em:

http://www.ncbi.nlm.nih.gov/pmc/articles/PMC3718499/

4. Conchon MF, Nascimento LA, Fonseca LF, Aroni P. Perioperative thirst: an analysis from the perspective of the Symptom Management Theory. Rev Esc Enferm USP [Internet]. 2015. [acesso em: 24 set. 2015]; 49(1):120-8.

Disponível em: http://www.ncbi.nlm.nih.gov/pubmed/25789651.

5. Silva LCJR, Aroni P, Fonseca LF. Tenho sede! Vivência do paciente cirúrgico no período perioperatório. Rev SOBECC [Internet]. 2016. [acesso em: 18 out. 2016]; 21(2); 75-81. Disponível em:

https://revista.sobecc.org.br/sobecc/article/view/21.

6. Gois CFL, Aguillar OM, Santos V, Rodríguez EOL. Fatores estressantes para o paciente submetido a cirurgia cardíaca. Invest Educ Enferm [Internet]. 2012. [acesso em: 6 set. 2015]; 30(3):312-9. Disponível em: http://www.scielo.org.co/scielo.php?pid=S0120-53072012000300003\&script=sci_arttext.

7. Dessotte CAM, Rodrigues HF, Furuya RK, Rossi LA, Danta RAS. Estressores percebidos por pacientes no pósoperatório imediato de cirurgia cardíaca. Rev Bras Enferm [Internet]. 2016 [acesso em: 14 out. 2016]; 69(4):741-50. Disponível em: http://www.scielo.br/pdf/reben/v69n4/0034-7167-reben-69-04-0741.pdf.

8. Kara B. Validity and Reliability of the Turkish version of the Thirst Distress Scale in patients on hemodialysis. Asian Nurs Res [Internet]. 2013 [acesso em: 20 out. 2016]; 7(4);212-8. Disponível em:

https://www.ncbi.nlm.nih.gov/pubmed/25030347.

9. Aroni $P$, Nascimento LA, Fonseca LF. Avaliação de estratégias no manejo da sede na sala de recuperação pósanestésica. Acta Paul Enferm [Internet]. 2012. [acesso em: 30 abr. 2015]; 24(4):530-6. Disponível em:

http://dx.doi.org/10.1590/S0103-21002012000400008.

10. Dodd M, Janson S, Facione N, Faucett J, Froelicher ES, Humphreys J et al. Advancing the science of symptom management. J Adv Nurs [Internet]. 2001 [acesso em: 5 nov. 2015]; 33(5):668-76. Disponível em:

http://www.ncbi.nlm.nih.gov/pubmed/11298204.

11. Egan G, Silk T, Zamarripa F, Williams J, Federico P, Cunnington R et al. Neural correlates of the emergence of consciousness of thirst. Proc Natl Acad Sci USA [Internet]. 2003 Dec [acesso em: 18 out. 2015]; 100(5):15241-6. Disponível em: http://www.pnas.org/content/100/25/15241.

12. Pavani MM, Fonseca LF, Conchon MF. Sede do paciente cirúrgico: percepções da equipe de enfermagem nas unidades de internação. Rev Enferm UFPE [Internet]. 2016 [acesso em: 20 out. 2016]; 10(9):3352-60. Disponível em: http://www.revista.ufpe.br/revistaenfermagem/index.php/revista/article/view/8084/pdf 11005.

13. Whittemore R, Knafl K. The integrative review: update methodology. J Adv Nurs [Internet]. 2005 [acesso em: 20 abr. 2015]; 29(4):337-41. Disponível em: http://www.ncbi.nlm.nih.gov/pubmed/16268861.

14. Bots CP, Brand HS, Veerman ECl, Korevaar JC, Valentijn-Benz M, Bezemer PD et al. Chewing gum and a saliva substitute alleviate thirst and xerostomia in patients on haemodialysis. Nephrol Dial Transplant [Internet]. 2005 [acesso em: 13 jun. 2015]; 20(3):578-84. Disponível em: http://www.ncbi.nlm.nih.gov/pubmed/15665029.

15. Bots CP, Brand HS, Poorterman JHG, van Amerongen BM, Valentijn-Benz M, Veerman ECl et al. Oral and salivary changes in patients with end stage renal disease (ESRD): a two year follow-up study.Br Dent J Transplant [Internet]. 2007 [acesso em: 6 maio 2015]; 202(2):1-5. Disponível em: http://www.ncbi.nlm.nih.gov/pubmed/17235362. 16. Fan WF, Zhang Q, Luo LH, Niu JY, Gu Y. Study on the clinical significance and related factors of thirst and xerostomia in maintenance hemodialysis patients. Kidney Blood Press Res [Internet]. 2013 [acesso em: 2 maio 2015]; 37(4-5):464-74. Disponível em: http://www.ncbi.nlm.nih.gov/pubmed/24247643.

17. Porcu M, Fanton E, Zampieron A. Thirst distress and interdialytic weight gain: a study on a sample of haemodialysis patients. J Ren Care [Internet]. 2007 [acesso em: 7 jun. 2015]; 33(4):179-81. Disponível em:

http://www.ncbi.nlm.nih.gov/pubmed/18298036.

18. Sung JM, Kuo SC, Guo HR, Chuang SF, Lee SY, Huang JJ. Decreased salivary flow rate as a dipsogenic factor in hemodialysis patients: evidence from an observational study and a pilocarpine clinical trial. J Am Soc Nephrol [Internet]. 2005 [acesso em: 15 jun. 2015]; 16(11):3418-29. Disponível em:

https://www.ncbi.nlm.nih.gov/pubmed/16177001.

19. Sung JM, Kuo SC, Guo HR, Chuang SF, Lee SY, Huang JJ. The role of oral dryness in interdialytic weight gain by

Rev. Eletr. Enf. [Internet]. 2017 [acesso em:_/_/_];19:a09. Disponível em: http://dx.doi.org/10.5216/ree.v19.40288. 
diabetic and non-diabetic haemodialysis patients. Nephrol Dial Transplant [Internet]. 2006 [acesso em: 15 jun. 2015]; 21(9):2521-28. Disponível em: http://www.ncbi.nlm.nih.gov/pubmed/16720594.

20. Yang LY, Yates P, Chin CC, Kao TK. Effect of acupressure on thirst in hemodialysis patients. Kidney Blood Press Res [Internet]. 2010 [acesso em: 25 jun. 2015]; 33(4):260-5. Disponível em:

http://www.ncbi.nlm.nih.gov/pubmed/20606475.

21. Li DT, Puntillo KA. A pilot study on coexisting symptoms in intensive care patients. Appl Nurs Res [Internet]. 2006 [acesso em: 4 dez. 2015]; 19(4):216-9. Disponível em: http://www.ncbi.nlm.nih.gov/pubmed/17098160.

22. Puntillo KA, Arai SR, Cohen NH, Gropper MA, Neuhaus J, Paul SM et al. Symptoms experienced by intensive care unit patients at high risk of dying. Crit Care Med [Internet]. 2010 [acesso em: 17 mar. 2015]; 38(11):2155-60.

Disponível em: http://www.ncbi.nlm.nih.gov/pubmed/20711069.

23. Puntillo KA, Arai SR, Cooper BA, Stotts NA, Nelson JE. A randomized clinical trial of an intervention to relieve thirst and dry mouth in intensive care unit patients. Intensive Care Med [Internet]. 2014 [acesso em: 17 mar. 2015]; 40(9):1295-1302. Disponível em: http://www.ncbi.nlm.nih.gov/pubmed/24894026.

24. Stotts NA, Arai SR, Cooper BA, Nelson JE, Puntillo KA. Predictors of thirst in intensive care unit patients. J Pain Symptom Manage [Internet]. 2015 [acesso em: 10 maio 2015]; 49(3):530-8. Disponível em:

http://www.ncbi.nlm.nih.gov/pubmed/25116914.

25. Ozoene JO, Enosolease ME, Ajayi OI, Agoreyo FO, Obika LFO. Thirst perception in dehydrated sickle cell disease patients in steady state. Niger J Physiol Sci [Internet]. 2009 [acesso em: 2 jun. 2015]; 24(2):121-7. Disponível em: https://www.ncbi.nlm.nih.gov/pubmed/16177001.

26. Siami S, Polito A, Porcher R, Hissem T, Blanchard A, Boucly C et al. Thirst perception and osmoregulation of vasopressin secreted are altered during recovery from septic shock. PLoSOne [Internet]. 2013 [acesso em: 5 maio 2015]; 8(11):1-7. Disponível em: http://www.ncbi.nlm.nih.gov/pubmed/24223220.

27. Farrel MJ, Zamarripa F, Shade R, Phillips PA, McKinley M, Fox PT et al. Effect of aging on regional cerebral flow responses associated with osmotic thirst and its saciation by water drinking: a PET study. Proced Natl Acad Sci USA [Internet]. 2008 [acesso em: 2 maio 2015]; 105(1):382-7. Disponível em:

http://www.pnas.org/content/105/1/382.abstract.

28.Pross N, Demazières A, Girard N, Barnouin R, Metzger D, Klein A et al. Effects of changes in water intake on mood of high and low drinkers. PLoS One [Internet]. 2014 [acesso em: 2 jun. 2015]; 9(4):1-7. Disponível em:

https://doi.org/10.1371/journal.pone.0094754.

29. Buehrer S, Hanke U, Klaghofer R, Fruehauf M, Weiss M, Schmitz A. Hunger and thirst numeric rating scales are not valid estimates for gastric content volumes: a prospective investigation in healthy children. Pediatr Anesth [Internet]. 2014 [acesso em: 6 maio 2015]; 24(3):309-15. Disponível em: http://www.ncbi.nlm.nih.gov/pubmed/24467570. 30.Klemetti S, Kinnunen I, Suominen T, Antila H, Vahlberg T, Grenman R et al. The effect of preoperative fasting on postoperative thirst, hunger and oral intake in paediatric ambulatory tonsillectomy. J Clin Nurs [Internet]. 2010 [acesso em: 15 maio 2015]; 19(3-4):341-50. Disponível em: http://www.ncbi.nlm.nih.gov/pubmed/20500273. 31. Cunha FF, Rêgo LP. Nursing and cancer pain. Rev Dor [Internet]. 2015 [acesso em: 2 nov. 2015]; 16(2):142-5. Disponível em: http://dx.doi.org/10.5935/1806-0013.20150027.

32. Ferreira-Valente MA, Pais-Ribeiro JL, Jensen MP. Validity of four pain intensity rating scales. Pain [Internet]. 2015 [acesso em: 2 nov. 2015]; 16(2):142-5. Disponível em: http://www.ncbi.nlm.nih.gov/pubmed/21856077.

33. Fortunato JGS, Furtado MS, Hirabae LFA, Oliveira JA. Escalas de dor no paciente crítico: uma revisão integrativa. Revista HUPE [Internet]. 2013 [acesso em: 6 nov. 2015]; 12(3):110-7. Disponível em:

http://revista.hupe.uerj.br/detalhe_artigo.asp?id=426.

34. Jensen MP. The validity and reliability of pain measures in adults with cancer. J Pain [Internet]. 2003 [acesso em: 5 nov. 2015]; 4(1):2-21. Disponível em: http://www.ncbi.nlm.nih.gov/pubmed/14622723.

35. Pereira LV, Pereira GA, Moura LA, Fernandes RR. Intensidade da dor em idosos institucionalizados: comparação entre as escalas numérica e de descritores verbais. Rev Esc Enferm USP [Internet]. 2015 [acesso em: 1ㅇ dez. 2015]; 49(5):804-10. Disponível em: http://www.revistas.usp.br/reeusp/article/view/106712.

36. Jaywant SS, Pai AVA. A comparative study of pain measurement scales in acute burn patients. Indian J Physiother Occup Ther [Internet]. 2003 [acesso em: 5 nov. 2015]; 35(3):13-7. Disponível em:

http://medind.nic.in/iba/t03/i3/ibat03i3p13.pdf.

37. Stevenson RJ, Mahmeet M, Rooney K. Individual differences in the interoceptive states of hunger, fullness and thirst. Appetite 2015 [acesso em: 10 dez. 2015]; 95 : 44-57. Disponível em:

Rev. Eletr. Enf. [Internet]. 2017 [acesso em:_/_/_];19:a09. Disponível em: http://dx.doi.org/10.5216/ree.v19.40288. 
https://www.ncbi.nlm.nih.gov/pubmed/26119812.

38. Thomson WM, Chalmers JM, Spencer AJ, Williams SM. The xerostomia inventory: a multi-item approach to measuring dry mouth. Community Dent Health [Internet]. 1999 [acesso em: 15 nov. 2015]; 16(1):12-7. Disponível em: https://www.ncbi.nlm.nih.gov/pubmed/10697349.

39. Bots CP, Brand HS, Veerman ECl, Korevaar JC, Valentijn-Benz M, van Amerongen BM et al. Interdialytic weight gain inpatients on hemodialysis is associated with dry mouth and thirst. Kidney Int [Internet]. 2004 [acesso em: 13 jun. 2015]; 66(4):1662-8. Disponível em: http://www.ncbi.nlm.nih.gov/pubmed/15458464.

40. Kolcaba KY. The art of comfort care. Image J Nurs Scholarsh [Internet]. 1995 [citado em 20 nov. 2015]; 27(4):287-9. Disponível em: https://www.ncbi.nlm.nih.gov/pubmed/8530116.

41. Welch JL. Development of the thirst distress scale. Nephrol Nurs J [Internet]. 2002 [acesso em: 4 abr. 2015]; 29(4):337-41. Disponível em: https://www.ncbi.nlm.nih.gov/pubmed/12224366.

42. Campana MC, Fonseca LF, Lopes DFM, Martins PR. Percepção dos cuidadores quanto à sede da criança cirúrgica. Rev Rene [Internet]. 2015 [acesso em: 20 out. 2016]; 16(6):799-808. Disponível em:

http://www.revistarene.ufc.br/revista/index.php/revista/article/view/2063. 